\title{
The diamond integral reverse Hölder inequality and related results on time scales
}

\author{
Guang-Sheng Chen ${ }^{1,2}$ and Cheng-Dong We $\mathrm{H}^{3^{*}}$ \\ Dedicated to Professor Ravi P Agarwal.
}

"Correspondence:

1612971951@qq.com

${ }^{3}$ School of Mathematics and

Statistics, Guangxi Teachers

Education University, Guangxi,

Nanning 530023, China

Full list of author information is

available at the end of the article

\begin{abstract}
In this paper, we establish reverse Hölder's inequality on time scales via diamond integral, which is defined as an 'approximate' symmetric integral on time scales. Moreover, we give some generalizations of diamond integral Hölder's inequality which is due to Brito da Cruz et al. Several other related inequalities are also presented.

MSC: 26D15;26E70

Keywords: diamond integral; time scales; Hölder's inequality; Minkowski's inequality; Dresher's inequality
\end{abstract}

\section{Introduction}

The famous inequality due to Hölder can be stated as follows (see $[1,2]$ ).

Theorem 1.1 (see [1, 2]) Let $f(x)>0, g(x)>0, p>1,1 / p+1 / q=1$. If $f(x)$ and $g(x)$ are continuous real-valued functions on $[a, b]$, then we have the following assertion:

$$
\int_{a}^{b} f(x) g(x) d x \leq\left(\int_{a}^{b} f^{p}(x) d x\right)^{1 / p}\left(\int_{a}^{b} g^{q}(x) d x\right)^{1 / q} .
$$

Hölder's inequality is of great interest in the qualitative theory of differential equations as well as other branches of mathematics.

In [3], the authors obtained the delta integral Hölder inequality on time scales as follows.

Theorem 1.2 Let $f, g, h \in C_{\mathrm{rd}}([a, b], \mathbb{R})$ and $1 / p+1 / q=1$ with $p>1$. Then we have the following assertion:

$$
\begin{aligned}
& \int_{a}^{b}|h(x)||f(x) g(x)| \Delta x \\
& \quad \leq\left(\int_{a}^{b}|h(x)||f(x)|^{p} \Delta x\right)^{\frac{1}{p}}\left(\int_{a}^{b}|h(x)||g(x)|^{q} \Delta x\right)^{\frac{1}{q}} .
\end{aligned}
$$

Nabla and diamond- $\alpha$ integral Hölder's inequality on time scales was established in [4], which can be demonstrated as follows.

(c) 2015 Chen and Wei. This article is distributed under the terms of the Creative Commons Attribution 4.0 International License (http://creativecommons.org/licenses/by/4.0/), which permits unrestricted use, distribution, and reproduction in any medium, provided you give appropriate credit to the original author(s) and the source, provide a link to the Creative Commons license, and indicate if changes were made. 
Theorem 1.3 Let $f, g, h \in C_{\mathrm{ld}}([a, b], \mathbb{R})$ and $1 / p+1 / q=1$ with $p>1$. Then we have the following assertion:

$$
\int_{a}^{b}|h(x)||f(x) g(x)| \nabla x \leq\left(\int_{a}^{b}|h(x)||f(x)|^{p} \nabla x\right)^{\frac{1}{p}}\left(\int_{a}^{b}|h(x)||g(x)|^{q} \nabla x\right)^{\frac{1}{q}} .
$$

Theorem 1.4 Let $f, g, h:[a, b] \rightarrow \mathbb{R}$ be $\diamond_{\alpha}$-integrable functions, and $1 / p+1 / q=1$ with $p>1$. Then we have the following assertion:

$$
\begin{aligned}
& \int_{a}^{b}|h(x)||f(x) g(x)| \diamond_{\alpha} x \\
& \quad \leq\left(\int_{a}^{b}|h(x)||f(x)|^{p} \diamond_{\alpha} x\right)^{\frac{1}{p}}\left(\int_{a}^{b}|h(x)||g(x)|^{q} \diamond_{\alpha} x\right)^{\frac{1}{q}} .
\end{aligned}
$$

Recently Anwar et al. [5] applied the theory of isotonic linear functionals to derive the following Hölder inequality.

Theorem 1.5 For $p>1$, define $q=p /(p-1)$. Let $E \subset \mathbb{R}^{n}$ be as in Theorem 3.7 in [5]. Assume that $|w||f|^{p},|w||g|^{q},|w f g|$ are $\Delta$-integrable on E. If $p>1$, then

$$
\int_{E}|w(t) f(t) g(t)| \Delta t \leq\left(\int_{E}|w(t)||f(t)|^{p} \Delta t\right)^{1 / p}\left(\int_{E}|w(t)||g(t)|^{q} \Delta t\right)^{1 / q}
$$

More recently Brito da Cruz et al. [6] introduced diamond integral Hölder's inequality on time scale as follows.

Theorem 1.6 Let $f, g: \mathbb{T} \rightarrow \mathbb{R}$ be $\diamond$-integrable on $[a, b]_{\mathbb{T}}, p>1$ with $1 / p+1 / q=1$. Then we have the following assertion:

$$
\int_{a}^{b}|f(x) g(x)| \diamond x \leq\left(\int_{a}^{b}|f(x)|^{p} \diamond x\right)^{1 / p}\left(\int_{a}^{b}|g(x)|^{q} \diamond x\right)^{1 / q}
$$

The purpose of this paper is to establish a reverse version and some generalizations of inequality (1.6). Some other related results are also considered. This paper is organized as follows. In Section 2, we introduce basic definitions and some preliminary results which are necessary in the sequel. Namely, we briefly introduce the nabla and the delta calculus $[7,8]$. We also present the notions of diamond- $\alpha$ integral and diamond integral which is defined as an 'approximate' symmetric integral on time scales, respectively [6, 9-11]; in Section 3, we give the main results; in Section 4, we establish some further generalizations and refinements of diamond integral Hölder's inequality; in Section 5, a subdividing of Hölder's inequality is obtained.

\section{Preliminaries}

A time scale $\mathbb{T}$ is an arbitrary nonempty closed subset of real numbers and has the topology that it inherits from the real numbers with the standard topology. Let $\mathbb{T}$ be a time scale. We consider two jump operators. The forward jump operator $\sigma: \mathbb{T} \rightarrow \mathbb{T}$ denoted by $\sigma(t):=\inf \{s \in \mathbb{T}: s>t\}$ with $\inf \emptyset=\sup \mathbb{T}($ i.e., $\sigma(M)=M$ if $\mathbb{T}$ has a maximum $M$ ); and the 
backward jump operator $\rho: \mathbb{T} \rightarrow \mathbb{T}$ denoted by $\sigma(t):=\sup \{s \in \mathbb{T}: s<t\}$ with $\sup \emptyset=\inf \mathbb{T}$ (i.e., $\rho(m)=m$ if $\mathbb{T}$ has a minimum $m$ ). Let

$$
\begin{aligned}
& \mathbb{T}^{\kappa}= \begin{cases}\mathbb{T} \backslash \sup \mathbb{T} & \text { if sup } \mathbb{T} \text { is finite and left-scattered, } \\
\mathbb{T}, & \text { otherwise, }\end{cases} \\
& \mathbb{T}_{\kappa}= \begin{cases}\mathbb{T} \backslash \inf \mathbb{T} & \text { if inf } \mathbb{T} \text { is finite and right-scattered, } \\
\mathbb{T}, & \text { otherwise. }\end{cases}
\end{aligned}
$$

We set $\mathbb{T}_{\kappa}^{\kappa}:=\mathbb{T}_{\kappa} \cap \mathbb{T}^{\kappa}$.

Definition 2.1 ([7]) Let $\mathbb{T}$ be a time-scale and $f: \mathbb{T} \rightarrow \mathbb{R} . f$ is delta differentiable at $t \in \mathbb{T}^{k}$ if there is a number $f^{\Delta}(t)$ with the property that for all $\varepsilon>0$, there exists a neighborhood $U$ of $t$ such that

$$
\left|f(\sigma(t))-f(s)-f^{\Delta}(t)(\sigma(t)-s)\right| \leq \varepsilon|\sigma(t)-s|
$$

for all $s \in U . f^{\Delta}(t)$ is called the delta derivative of $f$ at $t$. If $f$ is delta differentiable for all $t \in \mathbb{T}^{\kappa}$, then $f$ is said to be delta differentiable.

Definition $2.2([7])$ Let $\mathbb{T}$ be a time-scale and $f: \mathbb{T} \rightarrow \mathbb{R} . f$ is called nabla differentiable at $t \in \mathbb{T}_{\kappa}$ if there is a number $f^{\nabla}(t)$ with the property that for all $\varepsilon>0$, there exists a neighborhood $V$ of $t$ such that

$$
\left|f(\rho(t))-f(s)-f^{\nabla}(t)(\rho(t)-s)\right| \leq \varepsilon|\rho(t)-s|
$$

for all $s \in V \cdot f^{\nabla}(t)$ is called the nabla derivative of $f$ at $t$. If $f$ is nabla differentiable for all $t \in \mathbb{T}_{\kappa}$, then $f$ is nabla differentiable.

In what follows, assume that $a, b \in \mathbb{T}$ with $a<b,[a, b]_{\mathbb{T}}=\{t \in \mathbb{T}: a \leq t \leq b\}$.

Definition 2.3 ([7]) A function $F: \mathbb{T} \rightarrow \mathbb{R}$ is said to be a delta antiderivative of $f: \mathbb{T} \rightarrow \mathbb{R}$ if $F^{\Delta}(t)=f(t)$ holds true for all $t \in \mathbb{T}^{\kappa}$. We may define the delta integral of $f$ from $a$ to $b$ (or on $[a, b]_{\mathbb{T}}$ ) by

$$
\int_{a}^{b} f(t) \Delta t=F(b)-F(a)
$$

Definition 2.4 ([7]) A function $G: \mathbb{T} \rightarrow \mathbb{R}$ is said to be a nabla antiderivative of $g: \mathbb{T} \rightarrow \mathbb{R}$ if $G^{\nabla}(t)=g(t)$ holds true for all $t \in \mathbb{T}_{\kappa}$. We may define the nabla integral of $g$ from $a$ to $b$ (or on $[a, b]_{\mathbb{T}}$ ) by

$$
\int_{a}^{b} g(t) \nabla t=G(b)-G(a)
$$

For the properties of the delta and nabla integrals, please refer to $[7,8]$.

For a function $f: \mathbb{T} \rightarrow \mathbb{R}$, we define $f^{\sigma}(t)=f(\sigma(t))$ and $f^{\rho}(t)=f(\rho(t))$. 
Definition 2.5 ([10]) Assume that $t, s \in \mathbb{T}, \mu_{t, s}=\sigma(t)-s$ and $\eta_{t, s}=\rho(t)-s$. A function $f: \mathbb{T} \rightarrow \mathbb{R}$ is called diamond- $\alpha$ differentiable at $t \in \mathbb{T}_{\kappa}^{\kappa}$ if there is a number $f^{\diamond_{\alpha}}(t)$ with the property that for all $\varepsilon>0$, there exists a neighborhood $U$ of $t$ such that, for all $s \in U$,

$$
\left|\alpha\left[f^{\sigma}(t)-f(s)\right] \eta_{t, s}+(1-\alpha)\left[f^{\rho}(t)-f(s)\right] \mu_{t, s}-f^{\diamond_{\alpha}}(t) \mu_{t, s} \eta_{t, s}\right| \leq \varepsilon\left|\mu_{t, s} \eta_{t, s}\right| .
$$

If $f^{\diamond_{\alpha}}(t)$ exists for all $t \in \mathbb{T}_{\kappa}^{\kappa}$, then $f$ is called diamond- $\alpha$ differentiable.

Theorem 2.1 ([10]) Let $0 \leq \alpha \leq 1$. Iff is both nabla and delta differentiable at $t \in \mathbb{T}^{\kappa}$, then $f$ is diamond- $\alpha$ differentiable at $t$ and

$$
f^{\diamond_{\alpha}}(t)=\alpha f^{\Delta}(t)+(1-\alpha) f^{\nabla}(t)
$$

Remark 2.1 ([10]) If $\alpha=1$ in (2.1), then we obtain the delta derivative; if $\alpha=0$ in (2.1), then we obtain the nabla derivative.

Remark 2.2 The diamond- $\alpha$ derivative was defined by equality (2.1) in [9].

Definition 2.6 ([10]) Assume that $a, b \in \mathbb{T}, a<b, h: \mathbb{T} \rightarrow \mathbb{R}$ and $\alpha \in[0,1]$. We may define the diamond- $\alpha$ integral (or $\diamond_{\alpha}$-integral) of $h$ from $a$ to $b$ (or on $[a, b]_{\mathbb{T}}$ ) by

$$
\int_{a}^{b} h(t) \diamond_{\alpha} t=\alpha \int_{a}^{b} h(t) \Delta t+(1-\alpha) \int_{a}^{b} h(t) \nabla t
$$

where $h$ is both delta and nabla integrable on $[a, b]_{\mathbb{T}}$.

For related results concerning the diamond- $\alpha$ integral, please refer to [9-16] and the references therein.

In [17], the authors defined the real function $\gamma$ by

$$
\gamma(t):=\lim _{s \rightarrow t} \frac{\sigma(t)-s}{\sigma(t)+2 t-2 s-\rho(t)}
$$

The above function is very important in the definition of diamond integral (Definition 2.7). We see that $\gamma$ is well defined, $0 \leq \gamma(t) \leq 1$ for all $t \in \mathbb{T}$, and

$$
\gamma(t)= \begin{cases}\frac{1}{2} & \text { if } t \text { is dense } \\ \frac{\sigma(t)-t}{\sigma(t)-\rho(t)} & \text { if } t \text { is not dense. }\end{cases}
$$

Definition 2.7 (see [6]) Assume that $f: \mathbb{T} \rightarrow \mathbb{R}$ and $a, b \in \mathbb{T}, a<b$. We may define the diamond integral (or $\diamond$-integral) of $f$ from $a$ to $b$ (or on $[a, b]_{\mathbb{T}}$ ) by

$$
\int_{a}^{b} f(t) \diamond t=\int_{a}^{b} \gamma(t) f(t) \Delta t+\int_{a}^{b}(1-\gamma(t)) f(t) \nabla t,
$$

where $\gamma f$ is delta integrable and $(1-\gamma) f$ is nabla integrable on $[a, b]_{\mathbb{T}}$. The function $f$ is said to be diamond integrable (or $\diamond$-integrable) provided $f$ is $\diamond$-integrable on $[a, b]_{\mathbb{T}}$ for all $a, b \in \mathbb{T}$. 
The $\diamond$-integral has the following properties which are introduced in [6].

Theorem 2.2 (see [6]) Assume that $f, g: \mathbb{T} \rightarrow \mathbb{R}$ are $\diamond$-integrable on $[a, b]_{\mathbb{T}}$. Let $c \in[a, b]_{\mathbb{T}}$ and $\lambda \in \mathbb{R}$. Then the following assertions hold true.

1. $\int_{a}^{a} f(t) \diamond t=0$;

2. $\int_{a}^{b} f(t) \diamond t=\int_{a}^{c} f(t) \diamond t+\int_{c}^{b} f(t) \diamond t$

3. $\int_{a}^{b} f(t) \diamond t=-\int_{b}^{a} f(t) \diamond t$

4. $\lambda f$ is $\diamond$-integrable on $[a, b]_{\mathbb{T}}$ and $\int_{a}^{b} \lambda f(t) \diamond t=\lambda \int_{a}^{b} f(t) \diamond t$;

5. $f+g$ is $\diamond$-integrable on $[a, b]_{\mathbb{T}}$ and $\int_{a}^{b}(f+g)(t) \diamond t=\int_{a}^{b} f(t) \diamond t+\int_{a}^{b} g(t) \diamond t$;

6. $f g$ is $\diamond$-integrable on $[a, b]_{\mathbb{T}}$;

7. for $p>0,|f|^{p}$ is $\diamond$-integrable on $[a, b]_{\mathbb{T}}$;

8. iff $(t) \leq g(t)$ for all $t \in[a, b]_{\mathbb{T}}$, then $\int_{a}^{b} f(t) \diamond t \leq \int_{a}^{b} g(t) \diamond t$;

9. $|f|$ is $\diamond$-integrable on $[a, b]_{\mathbb{T}}$ and $\left|\int_{a}^{b} f(t) \diamond t\right| \leq \int_{a}^{b}|f(t)| \diamond t$.

For more properties of the $\diamond$-integral, please refer to [6].

\section{Main results}

In the section, we establish and prove our main results.

Theorem 3.1 (Diamond integral Hölder's inequality) Let $f, g, h: \mathbb{T} \rightarrow \mathbb{R}$ be $\diamond$-integrable on $[a, b]_{\mathbb{T}}, p>1$ with $q=p /(p-1)$. Then we have the following assertion:

$$
\int_{a}^{b}|h(x)||f(x) g(x)| \diamond x \leq\left(\int_{a}^{b}|h(x)||f(x)|^{p} \diamond x\right)^{1 / p}\left(\int_{a}^{b}|h(x)||g(x)|^{q} \diamond x\right)^{1 / q} .
$$

Proof This proof is the same as the proof of Theorem 4 in [6]. Therefore, we omit it here.

Remark 3.1 For $h(x)=1$ in Theorem 3.1, inequality (3.1) reduces to inequality (1.6).

Theorem 3.2 (Diamond integral reverse Hölder's inequality) Let $f, g, h: \mathbb{T} \rightarrow \mathbb{R}$ be $\diamond$-integrable on $[a, b]_{\mathbb{T}}, 0<p<1$ with $q=p /(p-1)$. If $g^{q}$ is $\diamond$-integrable on $[a, b]_{\mathbb{T}}$, then we have the following assertion:

$$
\int_{a}^{b}|h(x)||f(x) g(x)| \diamond x \geq\left(\int_{a}^{b}|h(x)||f(x)|^{p} \diamond x\right)^{1 / p}\left(\int_{a}^{b}|h(x)||g(x)|^{q} \diamond x\right)^{1 / q} .
$$

Proof Without loss of generality, we assume that

$$
\left(\int_{a}^{b}|h(x)||f(x)|^{p} \diamond x\right)^{1 / p}\left(\int_{a}^{b}|h(x)||g(x)|^{q} \diamond x\right)^{1 / q} \neq 0
$$

and let

$$
\xi(x)=|h(x)||f(x)|^{p} / \int_{a}^{b}|h(\tau)||f(\tau)|^{p} \diamond \tau
$$

and

$$
\gamma(x)=|h(x)||g(x)|^{q} / \int_{a}^{b}|h(\tau)||g(\tau)|^{q} \diamond \tau .
$$


Since both functions $\xi(x)$ and $\gamma(x)$ are $\diamond$-integrable on $[a, b]_{\mathbb{T}}$, applying the following reverse Young inequality (see [18])

$$
x^{\frac{1}{p}} y^{\frac{1}{q}} \geq \frac{1}{p} x+\frac{1}{q} y, \quad x, y>0,0<p<1,1 / p+1 / q=1
$$

with equality holds if and only if $x=y$, we have

$$
\begin{aligned}
& \int_{a}^{b} \frac{|h(x)|^{1 / p}|f(x)|}{\left(\int_{a}^{b}|h(\tau)||f(\tau)|^{p} \diamond \tau\right)^{1 / p}} \frac{|h(x)|^{1 / q}|g(x)|}{\left(\int_{a}^{b}|h(\tau)||g(\tau)|^{q} \diamond \tau\right)^{1 / q}} \diamond x \\
& \quad=\int_{a}^{b} \xi^{1 / p}(x) \gamma^{1 / q}(x) \diamond x \geq \int_{a}^{b}\left(\frac{\xi(x)}{p}+\frac{\gamma(x)}{q}\right) \diamond x \\
& \quad=\frac{1}{p} \int_{a}^{b}\left(\frac{|h(x)||f(x)|^{p}}{\int_{a}^{b}|h(\tau)||f(\tau)|^{p} \diamond \tau}\right) \diamond x+\frac{1}{q} \int_{a}^{b}\left(\frac{|h(x)||g(x)|^{q}}{\int_{a}^{b}|h(\tau)||g(\tau)|^{q} \diamond \tau}\right) \diamond x=1 .
\end{aligned}
$$

This leads to the desired inequality.

Remark 3.2 For $h(x)=1$ in Theorem 3.2, inequality (3.2) is a reverse version of inequality (1.6).

Combining Theorem 3.1 and Theorem 3.2, we can establish the following generalization.

Corollary 3.1 Let $h, f_{j}: \mathbb{T} \rightarrow \mathbb{R}, p_{j} \in \mathbb{R}, j=1,2, \ldots, m, \sum_{j=1}^{m} 1 / p_{j}=1$. If $h$ and $f_{j}$ are $\diamond$-integrable on $[a, b]_{\mathbb{T}}$, then the following assertions hold true.

(1) For $p_{j}>1$, we have

$$
\int_{a}^{b}|h(x)|\left|\prod_{j=1}^{m} f_{j}(x)\right| \diamond x \leq \prod_{j=1}^{m}\left(\int_{a}^{b}|h(x)|\left|f_{j}(x)\right|^{p_{j}} \diamond x\right)^{1 / p_{j}} .
$$

(2) For $0<p_{1}<1, p_{j}<0, j=2, \ldots, m, f_{j}^{p_{j}}$ are $\diamond$-integrable on $[a, b]_{\mathbb{T}}$, we have

$$
\int_{a}^{b}|h(x)|\left|\prod_{j=1}^{m} f_{j}(x)\right| \diamond x \geq \prod_{j=1}^{m}\left(\int_{a}^{b}|h(x)|\left|f_{j}(x)\right|^{p_{j}} \diamond x\right)^{1 / p_{j}} .
$$

Theorem 3.3 (Diamond integral Minkowski's inequality) Let $f, g, h: \mathbb{T} \rightarrow \mathbb{R}$, and $p>1$. If $f, g$ and $h$ are $\diamond$-integrable on $[a, b]_{\mathbb{T}}$, then we have the following assertion:

$$
\begin{aligned}
& \left(\int_{a}^{b}|h(x)||f(x)+g(x)|^{p} \diamond x\right)^{1 / p} \\
& \quad \leq\left(\int_{a}^{b}|h(x)||f(x)|^{p} \diamond x\right)^{1 / p}+\left(\int_{a}^{b}|h(x)||g(x)|^{p} \diamond x\right)^{1 / p} .
\end{aligned}
$$

Proof This proof is the same as the proof in Theorem 5 in [6]. So we omit it here.

Remark 3.3 For $h(x)=1$ in Theorem 3.3, inequality (3.5) reduces to Theorem 5 in [6]. 
Theorem 3.4 (Diamond integral reverse Minkowski's inequality) Let $f, g, h: \mathbb{T} \rightarrow \mathbb{R}$, and $0<p<1$. If $f, g$ and $h$ are $\diamond$-integrable on $[a, b]_{\mathbb{T}}$, then we have the following assertion:

$$
\begin{aligned}
& \left(\int_{a}^{b}|h(x)||f(x)+g(x)|^{p} \diamond x\right)^{1 / p} \\
& \quad \geq\left(\int_{a}^{b}|h(x)||f(x)|^{p} \diamond x\right)^{1 / p}+\left(\int_{a}^{b}|h(x)||g(x)|^{p} \diamond x\right)^{1 / p} .
\end{aligned}
$$

Proof For $0<p<1$, let $\frac{1}{s}=p, \frac{1}{t}=1-p, a_{k}=m_{k}^{p}, b_{k}=n_{k}^{1 / p-1}$, applying the following Hölder inequality [1]:

$$
\sum_{k=1}^{n} a_{k} b_{k} \leq\left(\sum_{k=1}^{n} a_{k}^{s}\right)^{1 / s}\left(\sum_{k=1}^{n} b_{k}^{t}\right)^{1 / t}, \quad s>1, \frac{1}{s}+\frac{1}{t}=1,
$$

we have

$$
\sum_{k=1}^{n} m_{k}^{p} n_{k}^{1 / p-1} \leq\left(\sum_{k=1}^{n} m_{k}\right)^{p}\left(\sum_{k=1}^{n} n_{k}^{1 / p}\right)^{1-p} .
$$

Let

$$
\begin{aligned}
& M=\int_{a}^{b}|h(x)||f(x)|^{p} \diamond x, \quad N=\int_{a}^{b}|h(x)||g(x)|^{p} \diamond x, \\
& W=\left(\int_{a}^{b}|h(x)||f(x)|^{p} \diamond x\right)^{1 / p}+\left(\int_{a}^{b}|h(x)||g(x)|^{p} \diamond x\right)^{1 / p}=M^{1 / p}+N^{1 / p} .
\end{aligned}
$$

Applying inequality (3.7), we have

$$
\begin{aligned}
W & =M^{1 / p}+N^{1 / p} \\
& =M^{1 / p-1} \int_{a}^{b}|h(x)||f(x)|^{p} \diamond x+N^{1 / p-1} \int_{a}^{b}|h(x)||g(x)|^{p} \diamond x \\
& =\int_{a}^{b}|h(x)|\left(|f(x)|^{p} M^{1 / p-1}+|g(x)|^{p} N^{1 / p-1}\right) \diamond x \\
& \leq \int_{a}^{b}|h(x)||f(x)+g(x)|^{p}\left(M^{1 / p}+N^{1 / p}\right)^{1-p} \diamond x \\
& =\int_{a}^{b}|h(x)||f(x)+g(x)|^{p} W^{1-p} \diamond x=W^{1-p} \int_{a}^{b}|h(x)| f(x)+\left.g(x)\right|^{p} \diamond x,
\end{aligned}
$$

from the above result, we immediately arrive at Minkowski's inequality and the theorem is completely proved.

From Theorem 3.3 and Theorem 3.4, the following generalization is obtained.

Corollary 3.2 Let $f_{j}, h: \mathbb{T} \rightarrow \mathbb{R}, j=1,2, \ldots, m$. If $f_{j}$ and $h$ are $\diamond$-integrable on $[a, b]_{\mathbb{T}}$, then the following assertions hold true. 
(1) For $p>1$, we have

$$
\left(\int_{a}^{b}|h(x)|\left|\sum_{j=1}^{m} f_{j}(x)\right|^{p} \diamond x\right)^{1 / p} \leq \sum_{j=1}^{m}\left(\int_{a}^{b}|h(x)|\left|f_{j}(x)\right|^{p} \diamond x\right)^{1 / p} .
$$

(2) For $0<p<1$, we have

$$
\left(\int_{a}^{b}|h(x)|\left|\sum_{j=1}^{m} f_{j}(x)\right|^{p} \diamond x\right)^{1 / p} \geq \sum_{j=1}^{m}\left(\int_{a}^{b}|h(x)|\left|f_{j}(x)\right|^{p} \diamond x\right)^{1 / p} .
$$

Next, we give an analogue of Corollary 3.2.

Corollary 3.3 Let $f_{j}, h: \mathbb{T} \rightarrow \mathbb{R}, j=1,2, \ldots, m$. If $f_{j}$ and $h$ are $\diamond$-integrable on $[a, b]_{\mathbb{T}}$, then the following assertions hold true.

(1) For $p>1$, we have

$$
\int_{a}^{b}|h(x)|\left(\sum_{j=1}^{m}\left|f_{j}(x)\right|\right)^{p} \diamond x \geq \sum_{j=1}^{m} \int_{a}^{b}|h(x)|\left|f_{j}(x)\right|^{p} \diamond x .
$$

(2) For $0<p<1$, we have

$$
\int_{a}^{b}|h(x)|\left(\sum_{j=1}^{m}\left|f_{j}(x)\right|\right)^{p} \diamond x \leq \sum_{j=1}^{m} \int_{a}^{b}|h(x)|\left|f_{j}(x)\right|^{p} \diamond x .
$$

Proof (1) For $p>1$, let $s=p, r=1$ in Jensen's inequality [1], we obtain the following inequality:

$$
\left|f_{1}(x)\right|+\left|f_{2}(x)\right|+\cdots+\left|f_{m}(x)\right| \geq\left(\left|f_{1}(x)\right|^{p}+\left|f_{2}(x)\right|^{p}+\cdots+\left|f_{m}(x)\right|^{p}\right)^{1 / p}
$$

from the above inequality, we obtain

$$
|h(x)|\left(\left|f_{1}(x)\right|+\left|f_{2}(x)\right|+\cdots+\left|f_{m}(x)\right|\right)^{p} \geq|h(x)|\left(\left|f_{1}(x)\right|^{p}+\left|f_{2}(x)\right|^{p}+\cdots+\left|f_{m}(x)\right|^{p}\right),
$$

by integrating the above inequality with respect to $x$, we obtain the desired result.

(2) For $0<p<1$, let $s=1, r=p$ in Jensen's inequality [1], we have

$$
\left|f_{1}(x)\right|+\left|f_{2}(x)\right|+\cdots+\left|f_{m}(x)\right| \leq\left(\left|f_{1}(x)\right|^{p}+\left|f_{2}(x)\right|^{p}+\cdots+\left|f_{m}(x)\right|^{p}\right)^{1 / p}
$$

it follows from the above inequality that

$$
|h(x)|\left(\left|f_{1}(x)\right|+\left|f_{2}(x)\right|+\cdots+\left|f_{m}(x)\right|\right)^{p} \leq|h(x)|\left(\left|f_{1}(x)\right|^{p}+\left|f_{2}(x)\right|^{p}+\cdots+\left|f_{m}(x)\right|^{p}\right),
$$

by integrating the above inequality with respect to $x$, the desired result is obtained.

Now, we establish some improvements of diamond integral Minkowski's inequality in the following theorem. 
Theorem 3.5 Letf $f, g, h: \mathbb{T} \rightarrow \mathbb{R}$ be $\diamond$-integrable on $[a, b]_{\mathbb{T}}, p>0, s, t \in \mathbb{R} \backslash\{0\}$, and $s \neq t$.

(1) Let $p, s, t \in \mathbb{R}$ be different such that $s, t>1$ and $(s-t) /(p-t)>1$. Then

$$
\begin{aligned}
\int_{a}^{b}|h(x)||f(x)+g(x)|^{p} \diamond x \\
\leq \\
\quad\left[\left(\int_{a}^{b}|h(x)||f(x)|^{s} \diamond x\right)^{\frac{1}{s}}+\left(\int_{a}^{b}|h(x)||g(x)|^{s} \diamond x\right)^{\frac{1}{s}}\right]^{s(p-t) /(s-t)} \\
\quad \times\left[\left(\int_{a}^{b}|h(x)||f(x)|^{t} \diamond x\right)^{\frac{1}{t}}+\left(\int_{a}^{b}|h(x)||g(x)|^{t} \diamond x\right)^{\frac{1}{t}}\right]^{t(s-p) /(s-t)} .
\end{aligned}
$$

(2) Let $p, s, t \in \mathbb{R}$ be different such that $0<s<1$ and $0<t<1$ and $(s-t) /(p-t)<1$. Then

$$
\begin{aligned}
& \int_{a}^{b}|h(x)||f(x)+g(x)|^{p} \diamond x \\
& \geq\left[\left(\int_{a}^{b}|h(x)||f(x)|^{s} \diamond x\right)^{\frac{1}{s}}+\left(\int_{a}^{b}|h(x)||g(x)|^{s} \diamond x\right)^{\frac{1}{s}}\right]^{s(p-t) /(s-t)} \\
& \quad \times\left[\left(\int_{a}^{b}|h(x)||f(x)|^{t} \diamond x\right)^{\frac{1}{t}}+\left(\int_{a}^{b}|h(x)||g(x)|^{t} \diamond x\right)^{\frac{1}{t}}\right]^{t(s-p) /(s-t)} .
\end{aligned}
$$

Proof (1) We have $(s-t) /(p-t)>1$, and

$$
\int_{a}^{b}|h(x)||f(x)+g(x)|^{p} \diamond x=\int_{a}^{b}|h(x)|\left(|f(x)+g(x)|^{s}\right)^{(p-t) /(s-t)}\left(|f(x)+g(x)|^{t}\right)^{(s-p) /(s-t)} \diamond x,
$$

by using Hölder's inequality (3.1) with indices $(s-t) /(p-t)$ and $(s-t) /(s-p)$, we have

$$
\begin{aligned}
& \int_{a}^{b}|h(x)||f(x)+g(x)|^{p} \diamond x \\
& \quad \leq\left(\int_{a}^{b}|h(x)||f(x)+g(x)|^{s} \diamond x\right)^{(p-t) /(s-t)}\left(\int_{a}^{b}|h(x)||f(x)+g(x)|^{t} \diamond x\right)^{(s-p) /(s-t)} .
\end{aligned}
$$

On the other hand, by using Minkowski's inequality (3.5) for $s>1$ and $t>1$, respectively, we can see that the following assertions hold true:

$$
\begin{aligned}
& \left(\int_{a}^{b}|h(x)||f(x)+g(x)|^{s} \diamond x\right)^{\frac{1}{s}} \\
& \quad \leq\left(\int_{a}^{b}|h(x)||f(x)|^{s} \diamond x\right)^{\frac{1}{s}}+\left(\int_{a}^{b}|h(x)||g(x)|^{s} \diamond x\right)^{\frac{1}{s}}
\end{aligned}
$$

and

$$
\begin{aligned}
& \left(\int_{a}^{b}|h(x)||f(x)+g(x)|^{t} \diamond x\right)^{\frac{1}{t}} \\
& \quad \leq\left(\int_{a}^{b}|h(x)||f(x)|^{t} \diamond x\right)^{\frac{1}{t}}+\left(\int_{a}^{b}|h(x)||g(x)|^{t} \diamond x\right)^{\frac{1}{t}} .
\end{aligned}
$$

From (3.14), (3.15) and (3.16), we obtain the desired result. 
(2) We have $(s-t) /(p-t)<1$ and

$$
\begin{aligned}
& \int_{a}^{b}|h(x)||f(x)+g(x)|^{p} \diamond x \\
& \quad=\int_{a}^{b}|h(x)|\left(|f(x)+g(x)|^{s}\right)^{(p-t) /(s-t)}\left(|f(x)+g(x)|^{t}\right)^{(s-p) /(s-t)} \diamond x,
\end{aligned}
$$

by using reverse Hölder's inequality (3.2) with indices $(s-t) /(p-t)$ and $(s-t) /(s-p)$, respectively, we have

$$
\begin{aligned}
& \int_{a}^{b}|h(x)||f(x)+g(x)|^{p} \diamond x \\
& \quad \geq\left(\int_{a}^{b}|h(x)||f(x)+g(x)|^{s} \diamond x\right)^{(p-t) /(s-t)}\left(\int_{a}^{b}|h(x)||f(x)+g(x)|^{t} \diamond x\right)^{(s-p) /(s-t)} .
\end{aligned}
$$

On the other hand, in view of reverse Minkowski's inequality (3.6) for the cases of $0<$ $s<1$ and $0<t<1$, we can see that the following assertions hold true:

$$
\begin{aligned}
& \left(\int_{a}^{b}|h(x)||f(x)+g(x)|^{s} \diamond x\right)^{\frac{1}{s}} \\
& \quad \geq\left(\int_{a}^{b}|h(x)||f(x)|^{s} \diamond x\right)^{\frac{1}{s}}+\left(\int_{a}^{b}|h(x)||g(x)|^{s} \diamond x\right)^{\frac{1}{s}}
\end{aligned}
$$

and

$$
\begin{aligned}
& \left(\int_{a}^{b}|h(x)||f(x)+g(x)|^{t} \diamond x\right)^{\frac{1}{t}} \\
& \quad \geq\left(\int_{a}^{b}|h(x)||f(x)|^{t} \diamond x\right)^{\frac{1}{t}}+\left(\int_{a}^{b}|h(x)||g(x)|^{t} \diamond x\right)^{\frac{1}{t}} .
\end{aligned}
$$

By (3.17), (3.18) and (3.19), we get the desired result.

\section{Remark 3.4}

(1) From Theorem 3.5, for $p>1$, letting $s=p+\varepsilon, t=p-\varepsilon$, when $p$, $s, t$ are different, $s, t>1$, and letting $\varepsilon \rightarrow 0$, we obtain (3.5).

(2) From Theorem 3.5, for $0<p<1$, letting $s=p+\varepsilon, t=p-\varepsilon$, when $p, s, t$ are different, $0<s, t<1$, and letting $\varepsilon \rightarrow 0$, we obtain (3.6).

Theorem 3.6 (Diamond integral Dresher's inequality) Letf, $g, h: \mathbb{T} \rightarrow \mathbb{R}$ and $0<r<1<p$. Iff, $g$ and $h$ are $\diamond$-integrable on $[a, b]_{\mathbb{T}}$, then we have the following assertion:

$$
\begin{aligned}
& \left(\frac{\int_{a}^{b}|h(x)||f(x)+g(x)|^{p} \diamond x}{\int_{a}^{b}|h(x)||f(x)+g(x)|^{r} \diamond x}\right)^{1 /(p-r)} \\
& \quad \leq\left(\frac{\int_{a}^{b}|h(x)||f(x)|^{p} \diamond x}{\int_{a}^{b}|h(x)||f(x)|^{r} \diamond x}\right)^{1 /(p-r)}+\left(\frac{\int_{a}^{b}|h(x)||g(x)|^{p} \diamond x}{\int_{a}^{b}|h(x)||g(x)|^{r} \diamond x}\right)^{1 /(p-r)} .
\end{aligned}
$$


Proof Based on $\diamond$-integral Hölder's inequality (3.1) and Minkowski's inequality (3.5), we have

$$
\begin{aligned}
& \left(\int_{a}^{b}|h(x)||f(x)+g(x)|^{p} \diamond x\right)^{1 /(p-r)} \\
& \leq\left(\left(\int_{a}^{b}|h(x)||f(x)|^{p} \diamond x\right)^{1 / p}+\left(\int_{a}^{b}|h(x)||g(x)|^{p} \diamond x\right)^{1 / p}\right)^{p /(p-r)} \\
& =\left(\left(\frac{\int_{a}^{b}|h(x)||f(x)|^{p} \diamond x}{\int_{a}^{b}|h(x)||f(x)|^{r} \diamond x}\right)^{1 / p}\left(\int_{a}^{b}|h(x)||f(x)|^{r} \diamond x\right)^{1 / p}\right. \\
& \left.\quad+\left(\frac{\int_{a}^{b}|h(x)||g(x)|^{p} \diamond x}{\int_{a}^{b}|h(x)||g(x)|^{r} \diamond x}\right)^{1 / p}\left(\int_{a}^{b}|h(x)||g(x)|^{r} \diamond x\right)^{1 / p}\right)^{p /(p-r)} \\
& \leq\left(\left(\frac{\int_{a}^{b}|h(x)||f(x)|^{p} \diamond x}{\int_{a}^{b}|h(x)||f(x)|^{r} \diamond x}\right)^{1 /(p-r)}+\left(\frac{\int_{a}^{b}|h(x)||g(x)|^{p} \diamond x}{\int_{a}^{b}|h(x)||g(x)|^{r} \diamond x}\right)^{1 /(p-r)}\right) \\
& \quad \times\left(\left(\int_{a}^{b}|h(x)||f(x)|^{r} \diamond x\right)^{1 / r}+\left(\int_{a}^{b}|h(x)||g(x)|^{r} \diamond x\right)^{1 / r}\right)^{r /(p-r)} .
\end{aligned}
$$

From Theorem 3.4, we get

$$
\begin{aligned}
& \left(\left(\int_{a}^{b}|h(x)||f(x)|^{r} \diamond x\right)^{1 / r}+\left(\int_{a}^{b}|h(x)||g(x)|^{r} \diamond x\right)^{1 / r}\right)^{r} \\
& \quad \leq \int_{a}^{b}|h(x)||f(x)+g(x)|^{r} \diamond x .
\end{aligned}
$$

From (3.21) and (3.22), we get (3.20).

Corollary 3.4 Let $f_{j}, h: \mathbb{T} \rightarrow \mathbb{R}, 0<r<1<p, j=1,2, \ldots, m$. If $\left|f_{j}\right|$ and $h$ are $\diamond$-integrable on $[a, b]_{\mathbb{T}}$, then we have the following assertion:

$$
\left(\frac{\int_{a}^{b}|h(x)|\left|\sum_{j=1}^{m} f_{j}(x)\right|^{p} \diamond x}{\int_{a}^{b}|h(x)|\left|\sum_{j=1}^{m} f_{j}(x)\right|^{r} \diamond x}\right)^{1 /(p-r)} \leq \sum_{j=1}^{m}\left(\frac{\int_{a}^{b}|h(x)|\left|f_{j}(x)\right|^{p} \diamond x}{\int_{a}^{b}|h(x)|\left|f_{j}(x)\right|^{r} \diamond x}\right)^{1 /(p-r)} .
$$

Theorem 3.7 (Diamond integral reverse Dresher's inequality) Let $f, g, h: \mathbb{T} \rightarrow \mathbb{R}$ and $p \leq 0 \leq r \leq 1$. If $f, g, f^{p}, g^{p}$ and $h$ are $\diamond$-integrable on $[a, b]_{\mathbb{T}}$, then we have the following assertion:

$$
\begin{aligned}
& \left(\frac{\int_{a}^{b}|h(x)||f(x)+g(x)|^{p} \diamond x}{\int_{a}^{b}|h(x)||f(x)+g(x)|^{r} \diamond x}\right)^{1 /(p-r)} \\
& \quad \geq\left(\frac{\int_{a}^{b}|h(x)||f(x)|^{p} \diamond x}{\int_{a}^{b}|h(x)||f(x)|^{r} \diamond x}\right)^{1 /(p-r)}+\left(\frac{\int_{a}^{b}|h(x)||g(x)|^{p} \diamond x}{\int_{a}^{b}|h(x)||g(x)|^{r} \diamond x}\right)^{1 /(p-r)} .
\end{aligned}
$$

Proof Let $\alpha_{1} \geq 0, \alpha_{2} \geq 0, \beta_{1}>0$, and $\beta_{2}>0$, and $-1<\lambda<0$, using the following Radon inequality (see[1]):

$$
\sum_{k=1}^{n} \frac{a_{k}^{p}}{b_{k}^{p-1}} \leq \frac{\left(\sum_{k=1}^{n} a_{k}\right)^{p}}{\left(\sum_{k=1}^{n} b_{k}\right)^{p-1}}, \quad a_{k} \geq 0, b_{k}>0,0<p<1,
$$


we have

$$
\frac{\alpha_{1}^{\lambda+1}}{\beta_{1}^{\lambda}}+\frac{\alpha_{2}^{\lambda+1}}{\beta_{2}^{\lambda}} \leq \frac{\left(\alpha_{1}+\alpha_{2}\right)^{\lambda+1}}{\left(\beta_{1}+\beta_{2}\right)^{\lambda}} .
$$

Let

$$
\begin{array}{ll}
\alpha_{1}=\left(\int_{a}^{b}|h(x)||f|^{p} \diamond x\right)^{1 / p}, & \beta_{1}=\left(\int_{a}^{b}|h(x)||f|^{r} \diamond x\right)^{1 / r}, \\
\alpha_{2}=\left(\int_{a}^{b}|h(x)||g|^{p} \diamond x\right)^{1 / p}, & \beta_{2}=\left(\int_{a}^{b}|h(x)||g|^{r} \diamond x\right)^{1 / r},
\end{array}
$$

and let $\lambda=\frac{r}{p-r}$, from (3.25) to (3.27), it follows that

$$
\begin{aligned}
\frac{\alpha_{1}^{\lambda+1}}{\beta_{1}^{\lambda}}+\frac{\alpha_{2}^{\lambda+1}}{\beta_{2}^{\lambda}} & =\frac{\left(\int_{a}^{b}|h(x)||f|^{p} \diamond x\right)^{(\lambda+1) / p}}{\left(\int_{a}^{b}|h(x)||f|^{r} \diamond x\right)^{\lambda / r}}+\frac{\left(\int_{a}^{b}|h(x)||g|^{p} \diamond x\right)^{(\lambda+1) / p}}{\left(\int_{a}^{b}|h(x)||g|^{r} \diamond x\right)^{\lambda / r}} \\
& =\left(\frac{\left.\int_{a}^{b}|h(x)| f\right|^{p} \diamond x}{\left.\int_{a}^{b}|h(x)| f\right|^{r} \diamond x}\right)^{1 /(p-r)}+\left(\frac{\int_{a}^{b}|h(x)||g|^{p} \diamond x}{\int_{a}^{b}|h(x)||g|^{r} \diamond x}\right)^{1 /(p-r)} \leq \frac{\left(\alpha_{1}+\alpha_{2}\right)^{\lambda+1}}{\left(\beta_{1}+\beta_{2}\right)^{\lambda}} \\
& =\frac{\left[\left(\left.\int_{a}^{b}|h(x)| f\right|^{p} \diamond x\right)^{1 / p}+\left(\int_{a}^{b}|h(x)||g|^{p} \diamond x\right)^{1 / p}\right]^{p /(p-r)}}{\left[\left(\left.\int_{a}^{b}|h(x)| f\right|^{r} \diamond x\right)^{1 / r}+\left(\int_{a}^{b}|h(x)||g|^{r} \diamond x\right)^{1 / r}\right]^{r /(p-r)}}
\end{aligned}
$$

Since $-1<\lambda=\frac{r}{p-r}<0$, we may assume $p<0<r$, and by Theorem 3.4 and $0<r \leq 1$, we obtain

$$
\left[\left(\int_{a}^{b}|h(x)||f|^{r} \diamond x\right)^{1 / r}+\left(\int_{a}^{b}|h(x)||g|^{r} \diamond x\right)^{1 / r}\right]^{r} \leq \int_{a}^{b}|h(x)||f+g|^{r} \diamond x .
$$

For $p<0$, by reverse Hölder's inequality [1], we obtain the reverse version of inequality (3.7) as follows:

$$
\sum_{k=1}^{n} m_{k}^{p} n_{k}^{1 / p-1} \geq\left(\sum_{k=1}^{n} m_{k}\right)^{p}\left(\sum_{k=1}^{n} n_{k}^{1 / p}\right)^{1-p} .
$$

Assume that $f(x)$ and $g(x)$ are nonzero, let $W, M, N$ be as in the proof of Theorem 3.4, from the above inequality, we have

$$
\begin{aligned}
W & =\left(\int_{a}^{b}|h(x)||f(x)|^{p} \diamond x\right)^{1 / p}+\left(\int_{a}^{b}|h(x)||g(x)|^{p} \diamond x\right)^{1 / p}=M^{1 / p}+N^{1 / p} \\
& =M^{1 / p-1} \int_{a}^{b}|h(x)||f(x)|^{p} \diamond x+N^{1 / p-1} \int_{a}^{b}|h(x)||g(x)|^{p} \diamond x \\
& =\int_{a}^{b}|h(x)|\left(|f(x)|^{p} M^{1 / p-1}+|g(x)|^{p} N^{1 / p-1}\right) \diamond x \\
& \geq \int_{a}^{b}|h(x)||f(x)+g(x)|^{p}\left(M^{1 / p}+N^{1 / p}\right)^{1-p} \diamond x \\
& =\int_{a}^{b}|h(x)||f(x)+g(x)|^{p} W^{1-p} \diamond x=W^{1-p} \int_{a}^{b}|h(x)||f(x)+g(x)|^{p} \diamond x .
\end{aligned}
$$


That is,

$$
W \geq W^{1-p} \int_{a}^{b}|h(x)||f(x)+g(x)|^{p} \diamond x .
$$

Hence, we have

$$
W^{p} \geq \int_{a}^{b}|h(x)||f(x)+g(x)|^{p} \diamond x .
$$

Based on the above inequality, we obtain

$$
\left[\left(\int_{a}^{b}|h(x)||f|^{p} \diamond x\right)^{1 / p}+\left(\int_{a}^{b}|h(x)||g|^{p} \diamond x\right)^{1 / p}\right]^{p} \geq \int_{a}^{b}|h(x)||f+g|^{p} \diamond x .
$$

From (3.28) to (3.30), we obtain reverse Dresher's inequality and the theorem is completely proved.

Corollary 3.5 Let $_{j}, h: \mathbb{T} \rightarrow \mathbb{R}, p \leq 0 \leq r<1, j=1,2, \ldots$, m. If $f_{j}, f_{j}^{p}$ and h are $\diamond$-integrable on $[a, b]_{\mathbb{T}}$, then we have the following assertion:

$$
\left(\frac{\int_{a}^{b}|h(x)|\left|\sum_{j=1}^{m} f_{j}(x)\right|^{p} \diamond x}{\int_{a}^{b}|h(x)|\left|\sum_{j=1}^{m} f_{j}(x)\right|^{r} \diamond x}\right)^{1 /(p-r)} \geq \sum_{j=1}^{m}\left(\frac{\int_{a}^{b}|h(x)|\left|f_{j}(x)\right|^{p} \diamond x}{\int_{a}^{b}|h(x)|\left|f_{j}(x)\right|^{r} \diamond x}\right)^{1 /(p-r)} .
$$

\section{Some further generalizations of Hölder's inequality}

The aim of this section is to derive some new generalizations and refinements of Hölder's inequality on time scales.

Theorem 4.1 Assume that $\mathbb{T}$ is a time scale, $a, b \in \mathbb{T}$ with $a<b$ and $p_{k}>0, \alpha_{k j} \in \mathbb{R}(j=$ $1,2, \ldots, m, k=1,2, \ldots, s), \sum_{k=1}^{s} \frac{1}{p_{k}}=1, \sum_{k=1}^{s} \alpha_{k j}=0, f_{j}, h: \mathbb{T} \rightarrow \mathbb{R}$. If $h$ and $f_{j}$ are $\diamond$-integrable on $[a, b]_{\mathbb{T}}$, then the following assertions hold true.

(1) For $p_{k}>1$, one has

$$
\int_{a}^{b}|h(x)|\left|\prod_{j=1}^{m} f_{j}(x)\right| \diamond x \leq \prod_{k=1}^{s}\left(\int_{a}^{b}|h(x)| \prod_{j=1}^{m}\left|f_{j}(x)\right|^{1+p_{k} \alpha_{k j}} \diamond x\right)^{1 / p_{k}} .
$$

(2) For $0<p_{s}<1, p_{k}<0(k=1,2, \ldots, s-1), f_{j}^{1+p_{k} \alpha_{k j}}$ is $\diamond$-integrable on $[a, b]_{\mathbb{T}}$, one has

$$
\int_{a}^{b}|h(x)|\left|\prod_{j=1}^{m} f_{j}(x)\right| \diamond x \geq \prod_{k=1}^{s}\left(\int_{a}^{b}|h(x)| \prod_{j=1}^{m}\left|f_{j}(x)\right|^{1+p_{k} \alpha_{k j}} \diamond x\right)^{1 / p_{k}} .
$$

Proof (1) Let

$$
g_{k}(x)=\left(\prod_{j=1}^{m} f_{j}^{1+p_{k} \alpha_{k j}}(x)\right)^{1 / p_{k}}
$$


Based on the assumptions $\sum_{k=1}^{s} \frac{1}{p_{k}}=1$ and $\sum_{k=1}^{s} \alpha_{k j}=0$, from a direct computation, it is obvious to show that

$$
\begin{aligned}
\prod_{k=1}^{s} g_{k}(x) & =g_{1}(x) g_{2}(x) \cdots g_{s}(x) \\
& =\left(\prod_{j=1}^{m} f_{j}^{1+p_{1} \alpha_{1 j}}(x)\right)^{1 / p_{1}}\left(\prod_{j=1}^{m} f_{j}^{1+p_{2} \alpha_{2 j}}(x)\right)^{1 / p_{2}} \cdots\left(\prod_{j=1}^{m} f_{j}^{1+p_{s} \alpha_{s j}}(x)\right)^{1 / p_{s}} \\
& =\prod_{j=1}^{m} f_{j}^{1 / p_{1}+\alpha_{1 j}}(x) \prod_{j=1}^{m} f_{j}^{1 / p_{2}+\alpha_{2 j}}(x) \cdots \prod_{j=1}^{m} f_{j}^{1 / p_{s}+\alpha_{s j}}(x) \\
& =\prod_{j=1}^{m} f_{j}^{1 / p_{1}+1 / p_{2}+\cdots+1 / p_{s}+\alpha_{1 j}+\alpha_{2 j}+\cdots+\alpha_{s j}}(x)=\prod_{j=1}^{m} f_{j}(x) .
\end{aligned}
$$

From the above result, we can obtain

$$
\prod_{k=1}^{s} g_{k}(x)=\prod_{j=1}^{m} f_{j}(x)
$$

Hence, we have

$$
\int_{a}^{b}|h(x)|\left|\prod_{j=1}^{m} f_{j}(x)\right| \diamond x=\int_{a}^{b}|h(x)|\left|\prod_{k=1}^{s} g_{k}(x)\right| \diamond x .
$$

It follows from Hölder's inequality (3.3) that

$$
\int_{a}^{b}|h(x)|\left|\prod_{k=1}^{s} g_{k}(x)\right| \diamond x \leq \prod_{k=1}^{s}\left(\int_{a}^{b}|h(x)|\left|g_{k}(x)\right|^{p_{k}} \diamond x\right)^{1 / p_{k}} .
$$

It follows from (4.3) and (4.5) that inequality (4.1) holds true.

(2) The proof of inequality (4.2) is similar to the proof of inequality (4.1), by (4.3), (4.4) and (3.4), we have

$$
\int_{a}^{b}|h(x)|\left|\prod_{k=1}^{s} g_{k}(x)\right| \diamond x \geq \prod_{k=1}^{s}\left(\int_{a}^{b}|h(x)|\left|g_{k}(x)\right|^{p_{k}} \diamond x\right)^{1 / p_{k}} .
$$

Based on (4.3) and (4.6), it follows that inequality (4.2) holds true.

Remark 4.1 Taking $s=m, \alpha_{k j}=-1 / p_{k}$ for $j \neq k$ and $\alpha_{k k}=1-1 / p_{k}$, inequalities (4.1) and (4.2) are respectively reduced to (3.3) and (3.4).

It is easy to see that many existing inequalities related to Hölder's inequality are special cases of inequalities (4.1) and (4.2). For example, we have the following.

Corollary 4.1 Under the assumptions of Theorem 4.1, taking $s=m, \alpha_{k j}=-t / p_{k}$ for $j \neq k$ and $\alpha_{k k}=t\left(1-1 / p_{k}\right)$ with $t \in \mathbb{R}$, the following assertions hold true. 
(1) For $p_{k}>1$, one has

$$
\begin{aligned}
& \int_{a}^{b}|h(x)|\left|\prod_{j=1}^{m} f_{j}(x)\right| \diamond x \\
& \quad \leq \prod_{k=1}^{m}\left(\int_{a}^{b}|h(x)|\left(\prod_{j=1}^{m}\left|f_{j}(x)\right|\right)^{1-t}\left(\left|f_{k}(x)\right|^{p_{k}}\right)^{t} \diamond x\right)^{1 / p_{k}} .
\end{aligned}
$$

(2) For $0<p_{m}<1, p_{k}<0(k=1,2, \ldots, m-1)$, one has

$$
\begin{aligned}
& \int_{a}^{b}|h(x)|\left|\prod_{j=1}^{m} f_{j}(x)\right| \diamond x \\
& \geq \prod_{k=1}^{m}\left(\int_{a}^{b}|h(x)|\left(\prod_{j=1}^{m}\left|f_{j}(x)\right|\right)^{1-t}\left(\left|f_{k}(x)\right|^{p_{k}}\right)^{t} \diamond x\right)^{1 / p_{k}} .
\end{aligned}
$$

Theorem 4.2 Assume that $\mathbb{T}$ is a time scale, $a, b \in \mathbb{T}$ with $a<b$ and $p_{k}>0, r \in \mathbb{R}$, $\alpha_{k j} \in \mathbb{R}(j=1,2, \ldots, m, k=1,2, \ldots, s), \sum_{k=1}^{s} \frac{1}{p_{k}}=r, \sum_{k=1}^{s} \alpha_{k j}=0, f_{j}, h: \mathbb{T} \rightarrow \mathbb{R}$. If $f_{j}$ and $h$ are $\diamond$-integrable on $[a, b]_{\mathbb{T}}$, then the following assertions hold true.

(1) For $r p_{k}>1$, one has

$$
\int_{a}^{b}|h(x)|\left|\prod_{j=1}^{m} f_{j}(x)\right| \diamond x \leq \prod_{k=1}^{s}\left(\int_{a}^{b}|h(x)| \prod_{j=1}^{m}\left|f_{j}(x)\right|^{1+r p_{k} \alpha_{k j}} \diamond x\right)^{1 / r p_{k}} .
$$

(2) For $0<r p_{s}<1, r p_{k}<0(k=1,2, \ldots, s-1), f_{j}^{1+r p_{k} \alpha_{k j}}$ is $\diamond$-integrable on $[a, b]_{\mathbb{T}}$, one has

$$
\int_{a}^{b}|h(x)|\left|\prod_{j=1}^{m} f_{j}(x)\right| \diamond x \geq \prod_{k=1}^{s}\left(\int_{a}^{b}|h(x)| \prod_{j=1}^{m}\left|f_{j}(x)\right|^{1+r p_{k} \alpha_{k j}} \diamond x\right)^{1 / r p_{k}} .
$$

Proof (1) Since $r p_{k}>1$ and $\sum_{k=1}^{s} \frac{1}{p_{k}}=r$, we get $\sum_{k=1}^{s} \frac{1}{r p_{k}}=1$. Then by (4.1) we immediately obtain inequality (4.9).

(2) Since $0<r p_{s}<1, r p_{k}<0$ and $\sum_{k=1}^{s} \frac{1}{p_{k}}=r$, we have $\sum_{k=1}^{s} \frac{1}{r p_{k}}=1$, by (4.2), we immediately have inequality (4.10). This completes the proof.

From Theorem 4.2, we obtain the following corollary.

Corollary 4.2 Under the assumptions of Theorem 4.2, and let $s=2, p_{1}=p, p_{2}=q, \alpha_{1 j}=$ $-\alpha_{2 j}=\alpha_{j}$, then the following assertions hold true.

(1) For $r p>1$, one has

$$
\begin{aligned}
& \int_{a}^{b}|h(x)|\left|\prod_{j=1}^{m} f_{j}(x)\right| \diamond x \\
& \quad \leq\left(\int_{a}^{b}|h(x)| \prod_{j=1}^{m}\left|f_{j}(x)\right|^{1+r p \alpha_{j}} \diamond x\right)^{1 / r p}\left(\int_{a}^{b}|h(x)| \prod_{j=1}^{m}\left|f_{j}(x)\right|^{1-r q \alpha_{j}} \diamond x\right)^{1 / r q} .
\end{aligned}
$$


(2) For $0<r p<1$, one has

$$
\begin{aligned}
& \int_{a}^{b}|h(x)|\left|\prod_{j=1}^{m} f_{j}(x)\right| \diamond x \\
& \quad \geq\left(\int_{a}^{b}|h(x)| \prod_{j=1}^{m}\left|f_{j}(x)\right|^{1+r p \alpha_{j}} \diamond x\right)^{1 / r p}\left(\int_{a}^{b}|h(x)| \prod_{j=1}^{m}\left|f_{j}(x)\right|^{1-r q \alpha_{j}} \diamond x\right)^{1 / r q} .
\end{aligned}
$$

Now we present a refinement of inequalities (4.9) and (4.10), respectively.

Theorem 4.3 Under the assumptions of Theorem 4.2, the following assertions hold true.

(1) For $r p_{k}>1$, one has

$$
\int_{a}^{b}|h(x)|\left|\prod_{j=1}^{m} f_{j}(x)\right| \diamond x \leq \varphi(c) \leq \prod_{k=1}^{s}\left(\int_{a}^{b}|h(x)| \prod_{j=1}^{m}\left|f_{j}(x)\right|^{1+r p_{k} \alpha_{k j}} \diamond x\right)^{1 / r p_{k}}
$$

where

$$
\varphi(c) \equiv \int_{a}^{c}|h(x)| \prod_{j=1}^{m}\left|f_{j}(x)\right| \diamond x+\prod_{k=1}^{s}\left(\int_{c}^{b}|h(x)| \prod_{j=1}^{m}\left|f_{j}(x)\right|^{1+r p_{k} \alpha_{k j}} \diamond x\right)^{1 / r p_{k}}
$$

is a nonincreasing function with $a \leq c \leq b$.

(2) For $0<r p_{s}<1$, one has

$$
\int_{a}^{b}|h(x)|\left|\prod_{j=1}^{m} f_{j}(x)\right| \diamond x \geq \varphi(c) \geq \prod_{k=1}^{s}\left(\int_{a}^{b}|h(x)| \prod_{j=1}^{m}\left|f_{j}(x)\right|^{1+r p_{k} \alpha_{k j}} \diamond x\right)^{1 / r p_{k}},
$$

where

$$
\varphi(c) \equiv \int_{a}^{c}|h(x)| \prod_{j=1}^{m}\left|f_{j}(x)\right| \diamond x+\prod_{k=1}^{s}\left(\int_{c}^{b}|h(x)| \prod_{j=1}^{m}\left|f_{j}(x)\right|^{1+r p_{k} \alpha_{k j}} \diamond x\right)^{1 / r p_{k}}
$$

is a nondecreasing function with $a \leq c \leq b$.

Proof (1) Let

$$
g_{k}(x)=\left(\prod_{j=1}^{m} f_{j}^{1+r p_{k} \alpha_{k j}}(x)\right)^{1 / r p_{k}}
$$

By rearrangement and the assumptions of Theorem 4.2, we can obtain

$$
\prod_{j=1}^{m} f_{j}(x)=\prod_{k=1}^{s} g_{k}(x)
$$

Next, we prove that $\varphi(c)$ is a nonincreasing function with $a \leq c \leq b$. Applying the following Hölder inequality [1]:

$$
\sum_{i=1}^{n} \prod_{k=1}^{s} a_{i k} \leq \prod_{k=1}^{s}\left(\sum_{i=1}^{n} a_{i k}^{q_{j}}\right)^{1 / q_{k}}, \quad q_{k}>1, \sum_{k=1}^{s} \frac{1}{q_{k}}=1
$$


we can obtain

$$
\begin{aligned}
\sum_{i=1}^{2} \prod_{k=1}^{s} a_{i k} & =\sum_{i=1}^{2} a_{i 1} a_{i 2} \cdots a_{i s} \\
& =a_{11} a_{12} \cdots a_{1 s}+a_{21} a_{22} \cdots a_{2 s} \\
& \leq\left(a_{11}^{r p_{1}}+a_{21}^{r p_{1}}\right)^{1 / r p_{1}}\left(a_{12}^{r p_{2}}+a_{22}^{r p_{2}}\right)^{1 / r p_{2}} \cdots\left(a_{1 s}^{r p_{s}}+a_{2 s}^{r p_{s}}\right)^{1 / r p_{s}} \\
& =\prod_{k=1}^{s}\left(\sum_{i=1}^{2} a_{i k}^{r p_{k}}\right)^{1 / r p_{k}},
\end{aligned}
$$

where $\sum_{k=1}^{s} \frac{1}{r p_{k}}=1, r p_{k}>1$.

Let

$$
a_{1 k}=\left(\int_{c_{1}}^{c_{2}}|h(x)|\left|g_{k}(x)\right|^{r p_{k}} \diamond x\right)^{1 / r p_{k}}, \quad k=1,2 \ldots, s
$$

and

$$
a_{2 k}=\left(\int_{c_{2}}^{b}|h(x)|\left|g_{k}(x)\right|^{r p_{k}} \diamond x\right)^{1 / r p_{k}}, \quad k=1,2 \ldots, s
$$

by inequality (4.15), we have

$$
\begin{aligned}
& \prod_{k=1}^{s}\left(\int_{c_{1}}^{c_{2}}|h(x)|\left|g_{k}(x)\right|^{r p_{k}} \diamond x\right)^{1 / r p_{k}}+\prod_{k=1}^{s}\left(\int_{c_{2}}^{b}|h(x)|\left|g_{k}(x)\right|^{r p_{k}} \diamond x\right)^{1 / r p_{k}} \\
& \quad \leq \prod_{k=1}^{s}\left(\int_{c_{1}}^{c_{2}}|h(x)|\left|g_{k}(x)\right|^{r p_{k}} \diamond x+\int_{c_{2}}^{b}|h(x)|\left|g_{k}(x)\right|^{r p_{k}} \diamond x\right)^{1 / r p_{k}} .
\end{aligned}
$$

Let $a \leq c_{1}<c_{2} \leq b$, by inequalities (3.3) and (4.16), we obtain

$$
\begin{aligned}
\varphi\left(c_{2}\right) \equiv & \int_{a}^{c_{2}}|h(x)| \prod_{j=1}^{m}\left|f_{j}(x)\right| \diamond x+\prod_{k=1}^{s}\left(\int_{c_{2}}^{b}|h(x)| \prod_{j=1}^{m}\left|f_{j}(x)\right|^{1+r p_{k} \alpha_{k j}} \diamond x\right)^{1 / r p_{k}} \\
= & \int_{a}^{c_{2}}|h(x)|\left|\prod_{k=1}^{s} g_{k}(x)\right| \diamond x+\prod_{k=1}^{s}\left(\int_{c_{2}}^{b}|h(x)|\left|g_{k}(x)\right|^{r p_{k}} \diamond x\right)^{1 / r p_{k}} \\
= & \int_{a}^{c_{1}}|h(x)|\left|\prod_{k=1}^{s} g_{k}(x)\right| \diamond x+\int_{c_{1}}^{c_{2}}|h(x)|\left|\prod_{k=1}^{s} g_{k}(x)\right| \diamond x \\
& +\prod_{k=1}^{s}\left(\int_{c_{2}}^{b}|h(x)|\left|g_{k}(x)\right|^{r p_{k}} \diamond x\right)^{1 / r p_{k}} \\
\leq & \int_{a}^{c_{1}}|h(x)|\left|\prod_{k=1}^{s} g_{k}(x)\right| \diamond x+\prod_{k=1}^{s}\left(\int_{c_{1}}^{c_{2}}|h(x)|\left|g_{k}(x)\right|^{r p_{k}} \diamond x\right)^{1 / r p_{k}} \\
& +\prod_{k=1}^{s}\left(\int_{c_{2}}^{b}|h(x)|\left|g_{k}(x)\right|^{r p_{k}} \diamond x\right)^{1 / r p_{k}}
\end{aligned}
$$




$$
\begin{aligned}
\leq & \int_{a}^{c_{1}}|h(x)|\left|\prod_{k=1}^{s} g_{k}(x)\right| \diamond x \\
& +\prod_{k=1}^{s}\left(\int_{c_{1}}^{c_{2}}|h(x)|\left|g_{k}(x)\right|^{r p_{k}} \diamond x+\int_{c_{2}}^{b}|h(x)|\left|g_{k}(x)\right|^{r p_{k}} \diamond x\right)^{1 / r p_{k}} \\
= & \int_{a}^{c_{1}}|h(x)|\left|\prod_{k=1}^{s} g_{k}(x)\right| \diamond x+\prod_{k=1}^{s}\left(\int_{c_{1}}^{b}|h(x)|\left|g_{k}(x)\right|^{r p_{k}} \diamond x\right)^{1 / r p_{k}} \\
= & \varphi\left(c_{1}\right),
\end{aligned}
$$

that is,

$$
\varphi\left(c_{2}\right) \leq \varphi\left(c_{1}\right)
$$

It follows from the above result that $\varphi(c)$ is a nonincreasing function with $a \leq c \leq b$. Hence $\varphi(b) \leq \varphi(c) \leq \varphi(a)$, we obtained the desired result.

(2) The proof of inequality (4.14) is similar to the proof of inequality (4.13), so we omit it here.

\section{A subdividing of diamond integral Hölder's inequality}

In this section, we give a subdividing of Hölder's inequality as follows.

Theorem 5.1 Let $f, g, h: \mathbb{T} \rightarrow \mathbb{R}$ be $\diamond$-integrable on $[a, b]_{\mathbb{T}}$, and $s, t \in \mathbb{R}$, and let $p=(s-$ $t) /(1-t), q=(s-t) /(s-1)$.

(1) If $s<1<t$ or $s>1>t$, then

$$
\begin{aligned}
& \int_{a}^{b}|h(x)||f(x) g(x)| \diamond x \\
& \leq\left(\int_{a}^{b}|h(x)||f(x)|^{s p} \diamond x\right)^{1 / p^{2}}\left(\int_{a}^{b}|h(x)||g(x)|^{t q} \diamond x\right)^{1 / q^{2}} \\
& \quad \times\left(\int_{a}^{b}|h(x)||f(x)|^{t p} \diamond x \int_{a}^{b}|h(x)||g(x)|^{s q} \diamond x\right)^{1 / p q} .
\end{aligned}
$$

(2) If $s>t>1$ or $s<t<1$; $t>s>1$ or $t<s<1$, then

$$
\begin{aligned}
& \int_{a}^{b}|h(x)||f(x) g(x)| \diamond x \\
& \geq\left(\int_{a}^{b}|h(x)||f(x)|^{s p} \diamond x\right)^{1 / p^{2}}\left(\int_{a}^{b}|h(x)||g(x)|^{t q} \diamond x\right)^{1 / q^{2}} \\
& \quad \times\left(\int_{a}^{b}|h(x)||f(x)|^{t p} \diamond x \int_{a}^{b}|h(x)||g(x)|^{s q} \diamond x\right)^{1 / p q} .
\end{aligned}
$$

Proof (1) Let $p=\frac{s-t}{1-t}$ and in view of $s<1<t$ or $s>1>t$, we have

$$
p=\frac{s-t}{1-t}>1
$$


by Hölder's inequality (3.1) with indices $\frac{s-t}{1-t}$ and $\frac{s-t}{s-1}$, we have

$$
\begin{aligned}
\int_{a}^{b}|h||f g| \diamond x & =\int_{a}^{b}|h||f g|^{s(1-t) /(s-t)}|f g|^{t(s-1) /(s-t)} \diamond x \\
& \leq\left(\int_{a}^{b}|h||f g|^{s} \diamond x\right)^{(1-t) /(s-t)}\left(\int_{a}^{b}|h||f g|^{t} \diamond x\right)^{(s-1) /(s-t)} .
\end{aligned}
$$

On the other hand, from Hölder's inequality again for $p=\frac{s-t}{1-t}>1$, it follows that the following two inequalities are true:

$$
\int_{a}^{b}|h||f g|^{s} \diamond x \leq\left(\int_{a}^{b}|h||f|^{s(s-t) /(1-t)} \diamond x\right)^{(1-t) /(s-t)}\left(\int_{a}^{b}|h||g|^{s(s-t) /(s-1)} \diamond x\right)^{(s-1) /(s-t)}
$$

and

$$
\begin{aligned}
\int_{a}^{b}|h||f g|^{t} \diamond x \leq & \left(\int_{a}^{b}|h||f|^{t(s-t) /(1-t)} \diamond x\right)^{(1-t) /(s-t)} \\
& \times\left(\int_{a}^{b}|h||g|^{t(s-t) /(s-1)} \diamond x\right)^{(s-1) /(s-t)} .
\end{aligned}
$$

From (5.3), (5.4) and (5.5), it follows that the case (1) of Theorem 5.1 holds true.

(2) Let $p=\frac{s-t}{1-t}$ and in view of $s>t>1$ or $s<t<1$, we have

$$
p=\frac{s-t}{1-t}<0
$$

and $t>s>1$ or $t<s<1$, we have $0<\frac{s-t}{1-t}<1$, by reverse Hölder's inequality (3.2) with indices $\frac{s-t}{1-t}$ and $\frac{s-t}{s-1}$, we have

$$
\begin{aligned}
\int_{a}^{b}|h||f g| \diamond x & =\int_{a}^{b}|h||f g|^{s(1-t) /(s-t)}|f g|^{t(s-1) /(s-t)} \diamond x \\
& \geq\left(\int_{a}^{b}|h||f g|^{s} \diamond x\right)^{(1-t) /(s-t)}\left(\int_{a}^{b}|h||f g|^{t} \diamond x\right)^{(s-1) /(s-t)} .
\end{aligned}
$$

On the other hand, from reverse Hölder's inequality again for $0<p=\frac{s-t}{1-t}<1$ or $p=\frac{s-t}{1-t}<$ 0 , it follows that the following two inequalities are true:

$$
\int_{a}^{b}|h||f g|^{s} \diamond x \geq\left(\int_{a}^{b}|h||f|^{s(s-t) /(1-t)} \diamond x\right)^{(1-t) /(s-t)}\left(\int_{a}^{b}|h||g|^{s(s-t) /(s-1)} \diamond x\right)^{(s-1) /(s-t)}
$$

and

$$
\begin{aligned}
\int_{a}^{b}|h||f g|^{t} \diamond x \geq & \left(\int_{a}^{b}|h||f|^{t(s-t) /(1-t)} \diamond x\right)^{(1-t) /(s-t)} \\
& \times\left(\int_{a}^{b}|h||g|^{t(s-t) /(s-1)} \diamond x\right)^{(s-1) /(s-t)} .
\end{aligned}
$$

From (5.6), (5.7) and (5.8), it follows that the case (2) of Theorem 5.1 holds true.

Remark 5.1 For $\mathbb{T}=\mathbb{R}$, Theorem 5.1 reduces to the results in [19]. 


\section{Competing interests}

The authors declare that they have no competing interests.

\section{Authors' contributions}

All authors contributed equally to the writing of this paper. All authors read and approved the final manuscript.

\section{Author details}

${ }^{1}$ Key Laboratory for Mixed and Missing Data Statistics of the Education Department of Guangxi Province, Guangxi Teachers Education University, Nanning, Guangxi 530023, China. ${ }^{2}$ Department of Construction and Information Engineering, Guangxi Modern Vocational Technology College, Hechi, Guangxi 547000, China. ${ }^{3}$ School of Mathematics and Statistics, Guangxi Teachers Education University, Guangxi, Nanning 530023, China.

\section{Acknowledgements}

The authors thank the editor and the referees for their valuable suggestions to improve the quality of this paper. This paper was partially supported by the Key Laboratory for Mixed and Missing Data Statistics of the Education Department of Guangxi Province (No. GXMMSL201404), the Scientific Research Project of Guangxi Education Department (Nos. YB2014560 and KY2015YB468) and the Natural Science Foundation of Guangxi Province (No. 2013JJAA10097).

Received: 28 October 2014 Accepted: 26 May 2015 Published online: 16 June 2015

\section{References}

1. Hardy, GH, Littlewood, JE, Pólya, G: Inequalities, 2nd edn. Cambridge University Press, Cambridge (1952)

2. Beckenbach, EF, Bellman, R: Inequalities. Springer, Berlin (1961)

3. Wong, F-H, Yeh, C-C, Lian, W-C: An extension of Jensen's inequality on time scales. Adv. Dyn. Syst. Appl. 1(1), 113-120 (2006)

4. Ozkan, UM, Sarikaya, MZ, Yildirim, H: Extensions of certain integral inequalities on time scales. Appl. Math. Lett. 21(10), 993-1000 (2008)

5. Anwar, M, Bibi, R, Bohner, M, Pečarić, J: Integral inequalities on time scales via the theory of isotonic linear functionals. Abstr. Appl. Anal. 2011, Article ID 483595 (2011). doi:10.1155/2011/483595

6. Brito da Cruz, AMC, Martins, N, Torres, DFM: The diamond integral on time scales. Bull. Malays. Math. Sci. Soc. (2014). doi:10.1007/s40840-014-0096-7

7. Bohner, M, Peterson, A: Dynamic Equations on Time Scales. Birkhäuser Boston, Boston (2001)

8. Kaymakcalan, B, Lakshimikantham, V, Sivasundaram, S: Dynamic Systems on Measure Chains. Kluwer Academic, Dordrecht (1996)

9. Sheng, Q, Fadag, M, Henderson, J, Davis, JM: An exploration of combined dynamic derivatives on time scales and their applications. Nonlinear Anal., Real World Appl. 7(3), 395-413 (2006)

10. Rogers, JW Jr., Sheng, Q: Notes on the diamond- $\alpha$ dynamic derivative on time scales. J. Math. Anal. Appl. 326(1), 228-241 (2007)

11. Sheng, Q: Hybrid approximations via second order combined dynamic derivatives on time scales. Electron. J. Qual. Theory Differ. Equ. 2007, 17 (2007)

12. Malinowska, AB, Torres, DFM: On the diamond-alpha Riemann integral and mean value theorems on time scales. Dyn. Syst. Appl. 18(3-4), 469-481 (2009)

13. Sidi Ammi, MR, Ferreira, RAC, Torres, DFM: Diamond- $\alpha$ Jensen's inequality on time scales. J. Inequal. Appl. 2008 Article ID 576876 (2008)

14. Mozyrska, D, Torres, DFM: Diamond-alpha polynomial series on time scales. Int. J. Math. Stat. 5(A09), 92-101 (2009)

15. Sidi Ammi, MR, Torres, DFM: Hölder's and Hardy's two dimensional diamond-alpha inequalities on time scales. An Univ. Craiova, Ser. Mat. Inform. 37(1), 1-11 (2010)

16. Brito da Cruz, AMC, Martins, N, Torres, DFM: Hahn's symmetric quantum variational calculus. Numer. Algebra Control Optim. 3(1), 77-94 (2013)

17. Brito da Cruz, AMC, Martins, N, Torres, DFM: Symmetric differentiation on time scales. Appl. Math. Lett. 26(2), 264-269 (2013)

18. Xing, J-S, Su, K-Q, Tao, P-F: The applications of Young inequality and Young inverse inequality. J. Zhoukou Norm. Univ. 24(2), 37-39 (2007) (in Chinese)

19. Zhao, C-J, Cheung, W-S: On subdividing of Hölder's inequality. Far East J. Math. Sci.: FJMS 60(1), 101-108 (2012) 